\title{
El clima durante el Plioceno en la región de Santa María AmajaC, HIDALGO, MÉXICO
}

\author{
Felisa J. Aguilar ${ }^{1}{ }^{2}$ y Ma. Patricia Velasco-de León ${ }^{1}$ \\ ${ }^{1}$ Facultad de Estudios Superiores Zaragoza, UN AM, Batallón 5 de Mayo s/n, Col. Ejército de 0 riente, Iztapalapa, \\ 09230, México, D. F. \\ "Laboratorio de Arqueozoología "M. en C. Ticul Álvarez Solórzano", Subdirección de Laboratorios y Apoyo \\ Académico, INAH, M oneda \# 16, Centro, 06060, M éxico, D. F. Correo electrónico: felisaaguilar@yahoo.com.mx
}

\begin{abstract}
Resumen: El clima en el que se desarrolló una comunidad vegetal en Santa María Amajac, Hidalgo, durante el Plioceno, fue inferido empleando el Climate Leaf Analysis Multivariate Program (CLAMP), el cual correlaciona la morfología foliar con el clima. Los parámetros meteorológicos estimados fueron: temperatura media anual: $16.7 \pm 1.2^{\circ} \mathrm{C}$; temperatura media para el mes más cálido: $26.4 \pm 1.6^{\circ} \mathrm{C}$; temperatura media para el mes más frío: $7^{\circ} \mathrm{C}$; duración de la estación de crecimiento: 9.2 meses; precipitación media en la estación de crecimiento: $72 \mathrm{~mm}$; precipitación de los tres meses más húmedos: $280 \mathrm{~mm}$; precipitación de los tres meses más secos: $62 \mathrm{~mm}$; precipitación media anual: $650 \pm 120 \mathrm{~mm}$; humedad relativa: $58 \%$; humedad específica: $7.8 \mathrm{~g} / \mathrm{kg}$; y entalpía: 309.5. A partir de estos valores, se sugiere que el clima correspondió a uno templado subhúmedo, $\mathrm{Ca}(\mathrm{w})\left(\mathrm{w}_{0}\right)\left(\mathrm{e}^{\prime}\right)$.

Palabras clave: CLAMP, fisonomía foliar, clima, Plioceno, Hidalgo, México

Abstract: The climate in which the Pliocene plant community of Santa María Amajac, Hidalgo, developed, was inferred using Climate Leaf Analysis Multivariate Program (CLAMP), which correlates leaf morphology with climate. The environmental parameters calculated were: mean annual temperature: $16.7 \pm 1.2^{\circ} \mathrm{C}$; warmest month mean temperature: $26.4 \pm 1.6^{\circ} \mathrm{C}$; coldest month mean temperature: $7^{\circ} \mathrm{C}$; growing season length: 9.2 months; mean growing season precipitation: $72 \mathrm{~mm}$; three wettest months: $280 \mathrm{~mm}$; three driest months: $62 \mathrm{~mm}$ : mean annual precipitation: $650 \pm 120 \mathrm{~mm}$; relative humidity: $58 \%$; specific humidity: $7.8 \mathrm{~g} / \mathrm{kg}$, and enthalpy: 309.5 . These values suggest that the climate was a subhumid, temperate one, $\mathrm{Ca}(\mathrm{w})\left(\mathrm{w}_{0}\right)\left(\mathrm{e}^{\prime}\right)$.

Keywords: CLAMP, leaf physiognomy, climate, Pliocene, Hidalgo, Mexico
\end{abstract}

E I clima es uno de los factores más importantes del ambiente, debido a que actúa sobre otros factores, modificándolos (García, 1986). El registro fósil indica que a lo largo de la historia de la Tierra han ocurrido variaciones climáticas a nivel mundial. Entre los procesos relacionados con estos cambios están las variaciones en el nivel del mar, los cambios en la topografía y el vulcanismo (Cavalier et al., 1981; Sear y Kelly, 1980). Las plantas constituyen uno de los grupos más utilizados para las interpretaciones paleoclimáticas, ya que éstas responden rápidamente a los cambios en las condiciones ambientales a las cuales están expuestas (Daubenmire, 1982). Uno de los órganos vegetativos de las plantas que responden a las fluctuaciones del clima son las hojas, ya que desarrollan características morfológicas eficaces para la realización de la fotosíntesis en el ambiente en el que se encuentran (Spicer, 1981; Wing y Sues, 1992). Entre los caracteres morfológicos de las hojas que varían al modificarse los parámetros climáticos se encuentran el tamaño, correlacionado con la humedad (Raunkier, 1934; Wolfe, 1993); el tipo de margen, correlacionado con la temperatura (Bailey y Sinnott, 1915; Wolfe, 1993; Wilf, 1997) y el tipo de ápice, correlacionado con la humedad (Wolfe, 1969, 1993).

Con base en la relación morfología foliar-clima, se desarrolló el método morfológico o fisonómico para establecer climas pretéritos usando a las hojas fósiles y así proponer cuáles climas existieron en diferentes regiones y edades geológicas (Wolfe y Hopkins, 1967; Wolfe, 1969, 1971; Dilcher, 1973; Dolph y Dilcher, 1979). Con este método se puede emplear un sólo carácter de la morfología foliar para determinar un parámetro climático por medio de un análisis univariado (Wilf, 1997; Wiemann et al., 1998), o varios caracteres foliares empleando análisis multivariados y prediciendo varios parámetros climáticos (Wiemann et al., 
1998); para este último caso, se han desarrollado diferentes bases de datos, con muestreos de la vegetación actual en diferentes partes del mundo, que han cuantificado los caracteres foliares de la vegetación y su relación con diferentes parámetros climáticos (Gregory-Wodzicki, 2000).

Una de estas bases es CLAMP (Climate Leaf Analysis Multivariate Program) (Wolfe, 1993), la cual utiliza ocho caracteres foliares y 31 estados de carácter, correlacionándolos con once parámetros meteorológicos (Wolfe, 1993, 2001; Herman et al., 1996; Spicer et al., 2002). CLAMP es una de las bases que toma en cuenta un mayor número de estados de carácter y variables meteorológicas, además de tener un mayor número de áreas muestreadas (Gregory-Wodzicki, 2000; Spicer et al., 2002) y de establecer como requisito para su empleo en una localidad fósil que al menos 20 especies o morfotipos se encuentren representados, ya que un número menor de 15 puede brindar estimaciones erróneas de los parámetros meteorológicos (Wolfe, 1993; Herman et al., 1996).

En México, los estudios de tipo ecológico en paleobotánica que empleen las hojas fósiles de angiospermas son muy escasos. Esto se debe a que los yacimientos de este tipo son poco conocidos en nuestro país, a que las hojas no son muy abundantes, a que no están bien preservadas, o a combinaciones de estas causas (González-Gallardo, 1987; Silva-Pineda y Buitrón-S., 2000), aunado al bajo número de paleobotánicos que existen en el país (Weber y CevallosFerris, 1997). Uno de los primeros estudios fue el realizado por Velasco-De León (1999) para plantas del Terciario en la Formación Pie de Vaca, en los alrededores de Tepexi de Rodríguez, Puebla, en donde se empleó CLAMP.

En el estado de Hidalgo existe una localidad fosilífera, Santa María Amajac, donde las hojas son abundantes, se encuentran bien preservadas, generalmente completas y representan un número de morfotipos superior al mínimo requerido por CLAMP. Por ello, el presente trabajo tuvo como finalidad inferir las variables meteorológicas de la paleocomunidad y proponer el clima que existió en la región durante el Plioceno.

\section{Área de estudio}

La localidad de Santa María Amajac se ubica aproximadamente a $4 \mathrm{~km}$ al sureste del poblado del mismo nombre, en las coordenadas geográficas $20^{\circ} 18^{\prime} 18^{\prime \prime} \mathrm{N} \mathrm{y}$ $98^{\circ} 42^{\prime} 44.4^{\prime \prime} \mathrm{O}$, y en coordenadas cartesianas a $1.9 \mathrm{~km}$ al $\mathrm{N} \mathrm{y}$ $4.6 \mathrm{~km}$ al $\mathrm{O}$ de Atotonilco el Grande, en el estado de Hidalgo, con una altitud de $1900 \mathrm{~m}$ (figura 1). La localidad se encuentra en la porción norte-centro de la provincia geológica Eje Neovolcánico, entre la Sierra de Pachuca y la Sierra Madre Oriental, y pertenece a la Formación Atotonilco el Grande (Beltrán-Romero y Luna-Gómez, 1994; Salvador-Flores, 2001), la cual comprende desde el Plioceno Tardío hasta el Pleistoceno Temprano (Segerstrom, 1961).

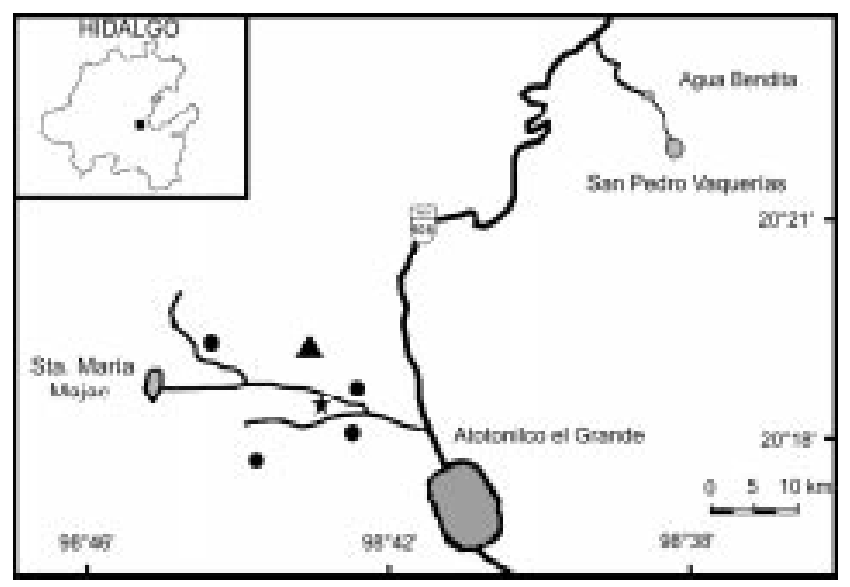

Figura 1. Ubicación de la localidad de Santa María Amajac (corresponde a la estrella) (Modificado de Castillo Cerón et al., 1996). Los círculos corresponden a las localidades reportadas por Castillo Cerón et al. (1996), el triángulo es la localidad de Santa Cruz de Amajac.

Descripción del afloramiento. El afloramiento estudiado tiene un espesor de $96.9 \mathrm{~m}$ y se caracteriza por presentar tres tipos de rocas: conglomerado, arenisca y lutita (figura 2). El conglomerado localizado en la parte superior tienen un espesor de $2 \mathrm{~m}$. La lutita y la arenisca se encuentran en capas intercaladas de espesor variable a lo largo del afloramiento, y en ellas se encuentran impresiones de hojas con diferente grado de preservación. Entre estas capas se observa una pequeña lámina de limonita que permite la preservación de algunos caracteres finos de las hojas (Salvador-Flores, 2001) (figura 2). Las hojas son abundantes, generalmente completas, aunque en algunos casos faltan principalmente los ápices y las de mayor tamaño se hallan fragmentadas.

Con respecto a la litología, Salvador-Flores (2001) indica que este afloramiento perteneció al margen de un paleolago presente en la región, y debido al tipo de estructuras sedimentarias (estratificación graduada, estratificación cruzada, gotas de lluvia y grietas de desecación) (figura 2), infiere que la zona estuvo sujeta a temporadas alternantes de humedad y de sequía.

Registros fosilíferos. En la región de Santa María Amajac se tienen diversos registros fósiles, que incluyen tanto elementos de fauna como de flora (cuadro 1). Además de Santa María Amajac, se tiene reporte de otras seis localidades con presencia de plantas fósiles; sin embargo, los autores no indican ni el estado de preservación ni la cantidad (cuadro 1).

Edad del afloramiento. El afloramiento no ha sido datado radiométricamente. Sin embargo, por la fauna encontrada en la región, principalmente las especies Equus simplicidens, Rhynchotherium sp. y Tremarctus sp. (Castillo-Cerón, 1994; 
El clima durante el Plioceno en Hidalgo, México

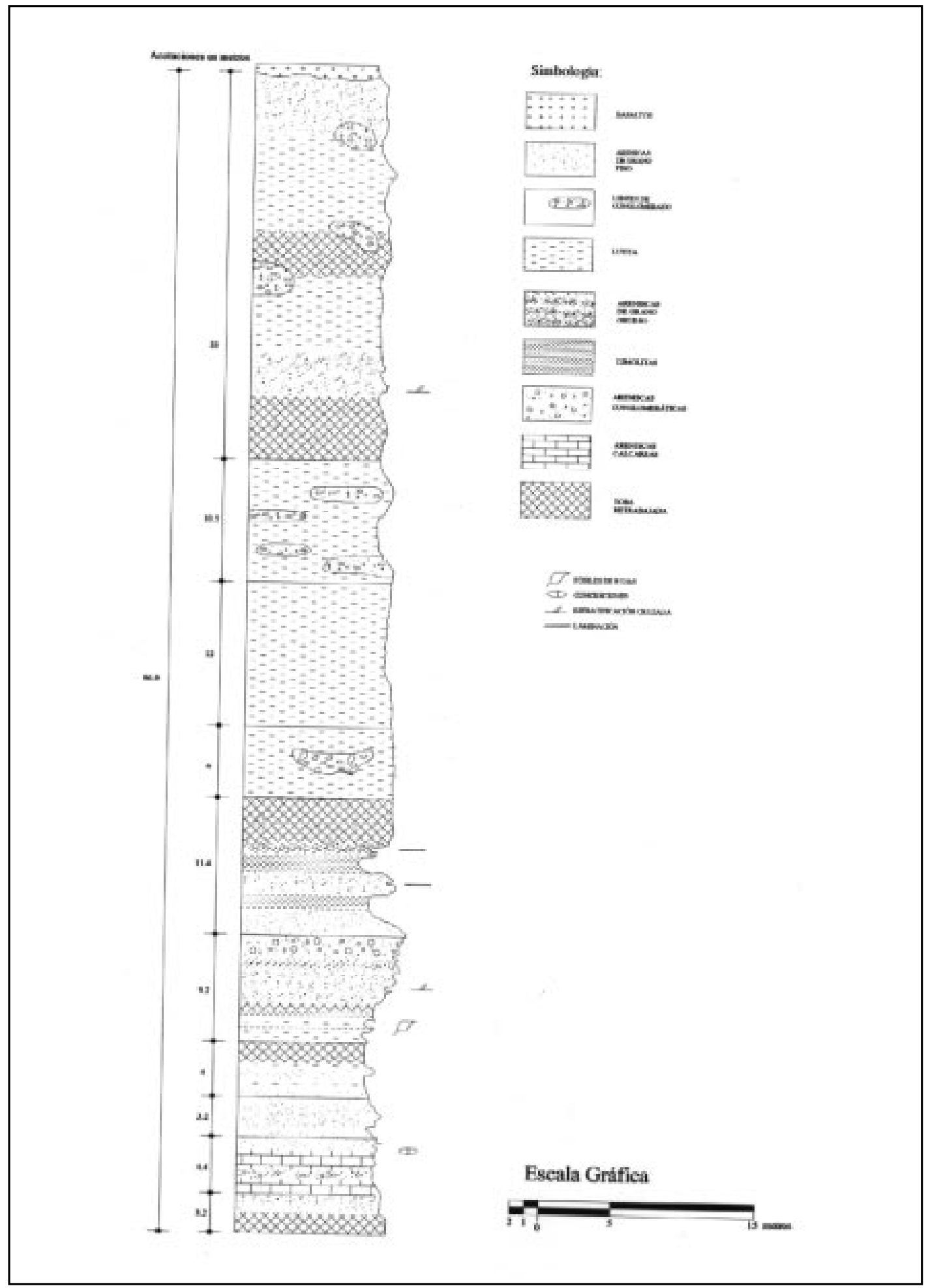

Figura 2. Columna geológica del afloramiento (tomada de Salvador-Flores, 2001). 
Cuadro 1. Registros previos de fósiles para la región de Santa M aría Amajac.

\begin{tabular}{|c|c|c|}
\hline Referencias & Localidad & Taxa reportados \\
\hline Villarello y Böse (1902) & Mina Transvaal & $\begin{array}{l}\text { Mastodon shepardi? } \\
\text { Equus excelsus } \\
\text { Elephas primigenius }\end{array}$ \\
\hline $\begin{array}{l}\text { Freudenberg (1922) } \\
\text { Miller (1987), Polaco et al. (2001) }\end{array}$ & $\begin{array}{l}\text { Valle de Amajac } \\
\text { Arroyo Amajac }\end{array}$ & $\begin{array}{l}\text { Mastodon oligobunis var. antiquissima } \\
\text { Mammut americanum }\end{array}$ \\
\hline $\begin{array}{l}\text { Beltrán-Romero y } \\
\text { Luna-Gómez (1994) }\end{array}$ & $\begin{array}{l}\text { Sureste del poblado El Paso de Amajac } \\
\text { Sanctorum }\end{array}$ & $\begin{array}{l}\text { Angiosperma } \\
\text { Planorbis sp. }\end{array}$ \\
\hline Castillo Cerón (1994) & $\begin{array}{l}\text { La Pinta de Vaquerías } \\
\text { Santa Cruz de Amajac } \\
\text { La M oneda } \\
\text { El Frontón } \\
\text { Puerta de las Lajas }\end{array}$ & Restos de vertebrados \\
\hline Castillo Cerón et al. (1996) & $\begin{array}{l}\text { La Pinta de Vaquerías } \\
\text { Santa Cruz de Amajac* } \\
\text { La Moneda* } \\
\text { El Frontón* } \\
\text { Puerta de las Lajas* } \\
\text { La Curva* }\end{array}$ & $\begin{array}{l}\text { Restos de vertebrados e impresiones } \\
\text { de plantas en las localidades } \\
\text { con asterisco }(*)\end{array}$ \\
\hline Carranza-Castañeda y M iller (1998) & Área de Santa M aría Amajac & $\begin{array}{l}\text { Equus simplicidens } \\
\text { Rhynchoterium sp. } \\
\text { Platygonus sp. }\end{array}$ \\
\hline \multicolumn{3}{|c|}{$\begin{array}{l}\text { Nota: la nomenclatura y la taxonomía de los taxa reportados se presenta como se encuentra en el trabajo original, es decir, no se } \\
\text { realizó ninguna actualización. }\end{array}$} \\
\hline
\end{tabular}

Carranza-Castañeda y Miller, 1998), las dataciones radiométricas realizadas en Santa Cruz de Amajac que le asignan una edad de $4.2 \pm 0.3$ millones de años (por la técnica de fission track) y de $4.57 \pm 0.02$ millones de años (con isótopos de argón $\left[{ }^{40} \mathrm{Ar} /{ }^{39} \mathrm{Ar}\right]$ ) (Kowallis et al., 1998), y el hecho de que Castillo-Cerón (1994) reconozca que toda la región de Santa María Amajac pertenece a la misma unidad litológica (figura 1), permiten asignar una edad tentativa para el afloramiento del Plioceno.

\section{Materiales y métodos}

El estudio se realizó con 525 ejemplares recolectados desde 1996 hasta 1998, mismos que se encuentran depositados en la colección paleontológica de la Facultad de Estudios Superiores Zaragoza, Universidad Nacional Autónoma de México (CFZ). Para la inferencia de las variables meteorológicas se utilizó el método fisonómico, empleando CLAMP. Para ello se realizó el registro de los caracteres y sus estados siguiendo a Wolfe (1993) y Herman et al. (1996) (figura 3), con excepción del tamaño de la hoja, donde se consideró la longitud desde la base hasta el ápice, para los 39 morfotipos presentes en la muestra de estudio (AguilarArellano y Ortíz-Martínez, 2000) (apéndice 1). Los datos obtenidos se codificaron y se calcularon los porcentajes correspondientes a cada estado de carácter para la muestra (apéndice 1) (Wolfe, 1993; Herman et al., 1996; Spicer et al., 2002), anexándolos a la versión CLAMP3B (Wolfe, 1995), utilizada en este trabajo.

La correlación de los parámetros climáticos y la fisonomía foliar en CLAMP se obtiene a través de un análisis canónico de correspondencia (ACC) (Kovach y Spicer, 1995; Herman et al., 1996; Gregory-Wodzicki, 2000; Wolfe, 2001), el cual ordena los sitios simultáneamente en un espacio multidimensional, como resultado de los caracteres fisonómicos de la comunidad y los datos meteorológicos, por medio de regresiones polinomiales de segundo grado (Kovach y Spicer, 1995; Herman y Spicer, 1996; Herman et al., 1996). Con base en esta calibración, Herman et al. (1996) desarrollaron ecuaciones para obtener los parámetros meteorológicos de una localidad fósil. Los parámetros meteorológicos estimados son: temperatura media anual (TMA), temperatura media del mes más cálido (TMC), temperatura media del mes más frío (TMF), duración de la estación de crecimiento (LEC), precipitación media anual (PMA), precipitación media en la estación de crecimiento (PMEC), precipitación de los tres meses más húmedos (3MH), precipitación de los tres meses más secos (3MS), 


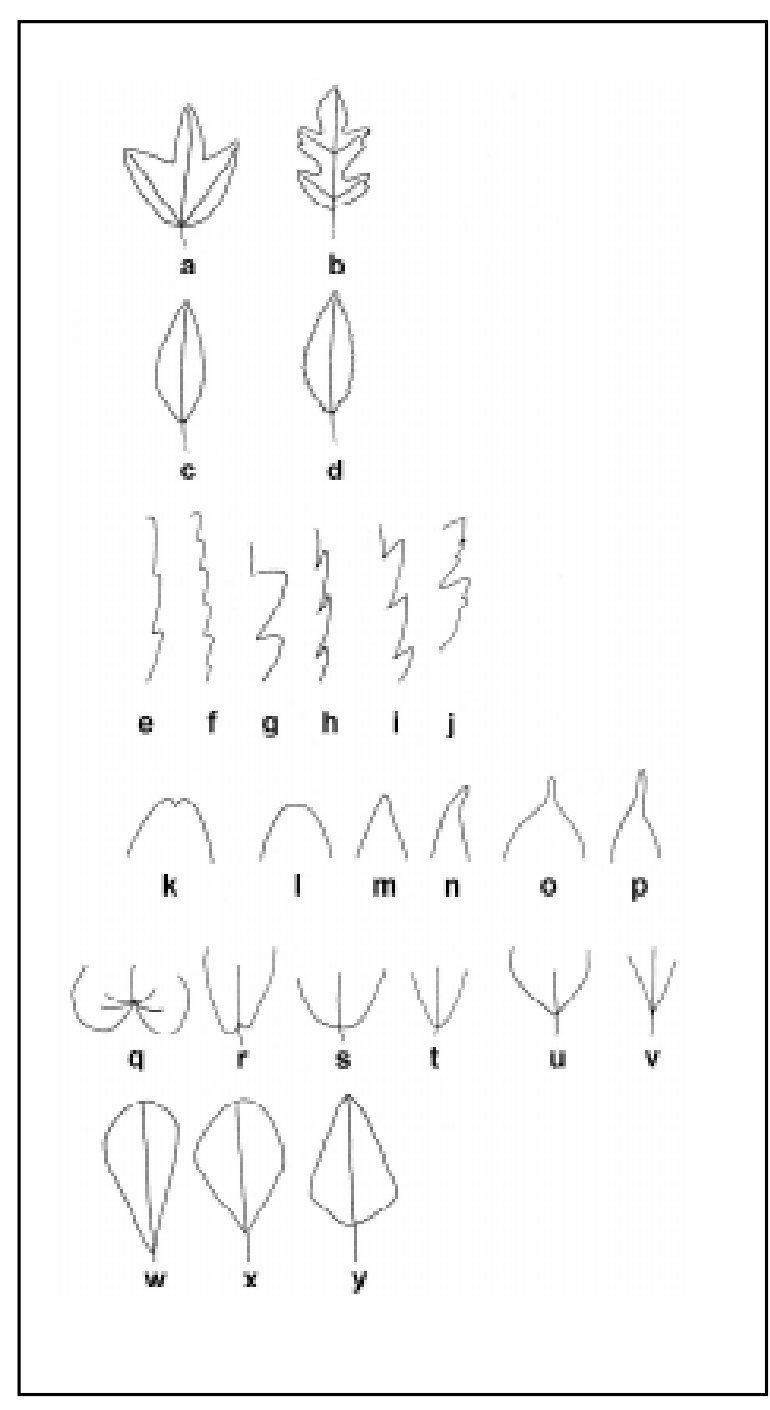

Figura 3. Caracteres y estados de carácter utilizados en CLAMP: hoja con lóbulos, a) palmado-lobada o b) pinado-lobada; margen, c) entero, d) dentado; tipo de diente, e) diente con espaciamiento regular, f) diente con espaciamiento cerrado, g) diente redondo, h) diente apresado, i) diente agudo, j) diente compuesto; tipo de ápice, k) emarginado, l) redondo, m-n) agudo, o-p) atenuado; tipo de base, q-r) cordada, s-t) redonda, $\mathrm{u}-\mathrm{v}$ ) aguda; forma, w) obovada, $\mathrm{x}$ ) elíptica, y) ovada. (Modificado de Herman et al., 1996). humedad relativa (HR), humedad específica (HE) y entalpía (ENT).

El ACC se realizó con el programa CANOCO (Ter Braak, 1991) y los cálculos de las ecuaciones de los parámetros meteorológicos de la comunidad fósil se hicieron con el programa EXCEL versión 5.0 (Microsoft Corporation, 1994).

\section{Resultados}

La fisonomía foliar de los 39 morfotipos reconocidos de la paleocomunidad de Santa María Amajac presenta los siguientes estados de carácter: hojas lobuladas, específicamente una palmado-lobado (figura 4, c); margen entero y dentado (figura 4, a, b, d y g), de este último, los dientes presentaban un espaciamiento regular (figura 4 , a y g); tamaño de las hojas de nanófilo a micrófilo 3; ápices agudos y redondos; bases cordadas, redondas y agudas; la relación largo-ancho oscila entre $<1$ ( $>4: 1$, la relación 3 ( 4:1 no se encuentra representada; las formas de las láminas son obovadas, elípticas y ovadas. De estos estados de carácter, los que tienen una mayor representatividad en la paleocomunidad son el margen entero $(72 \%)$, el tamaño micrófilo $2(56 \%)$, el ápice y la base aguda (68 y 54\%, respectivamente), la relación largo-ancho 1 ( $2: 1$ (74\%) y la forma de la lámina elíptica (54\%) (apéndice 1).

Los parámetros meteorológicos calculados para la localidad de estudio fueron: TMA de $16.7 \pm 1.2^{\circ} \mathrm{C}$ (Herman et al., 1996), TMC de $26.4 \pm 1.6^{\circ} \mathrm{C}$ (Wolfe, 2001), TMF de $7^{\circ} \mathrm{C}$, LEC de 9.2 meses, PMA de $650 \pm 120 \mathrm{~mm}$ (Wolfe, 1995), PMEC de $72 \mathrm{~mm}, 3 \mathrm{MH}$ de $280 \mathrm{~mm}, 3 \mathrm{MS}$ de $62 \mathrm{~mm}$, HR $58 \%$, HE $7.8 \mathrm{~g} / \mathrm{kg}$ y ENT 309.5 .

\section{Discusión}

El análisis de la fisonomía foliar indica que las condiciones de temperatura para la región eran algo bajas, debido a la predominancia del margen entero y el tamaño micrófilo 2 (apéndice 1), ya que éstos han sido asociados a intervalos de temperatura de $13-20^{\circ} \mathrm{C}$ (Wolfe, 1993). En cuanto a las condiciones de humedad, ésta era alta, ya que la forma elíptica, el ápice y la base agudos, y la relación largo-ancho 1-2:1 reflejan este tipo de condiciones; sin embargo, es posible que éstas no hayan sido constantes a lo largo del año, debido a que el tamaño micrófilo está asociado a la estacionalidad de la humedad, aspecto que se ve reflejado en la localidad por las estructuras sedimentarias presentes en el afloramiento (Salvador-Flores, 2001).

Inferencia del paleoclima. Para cuantificar la temperatura y la humedad de la localidad se utilizó CLAMP, ya que éste hace uso de un amplio número de caracteres y estados, con lo que se toma en cuenta la interacción entre la temperatura y la precipitación (Wolfe, 1995), además de que algunos caracteres pueden servir de amortiguador al reducir el efecto 


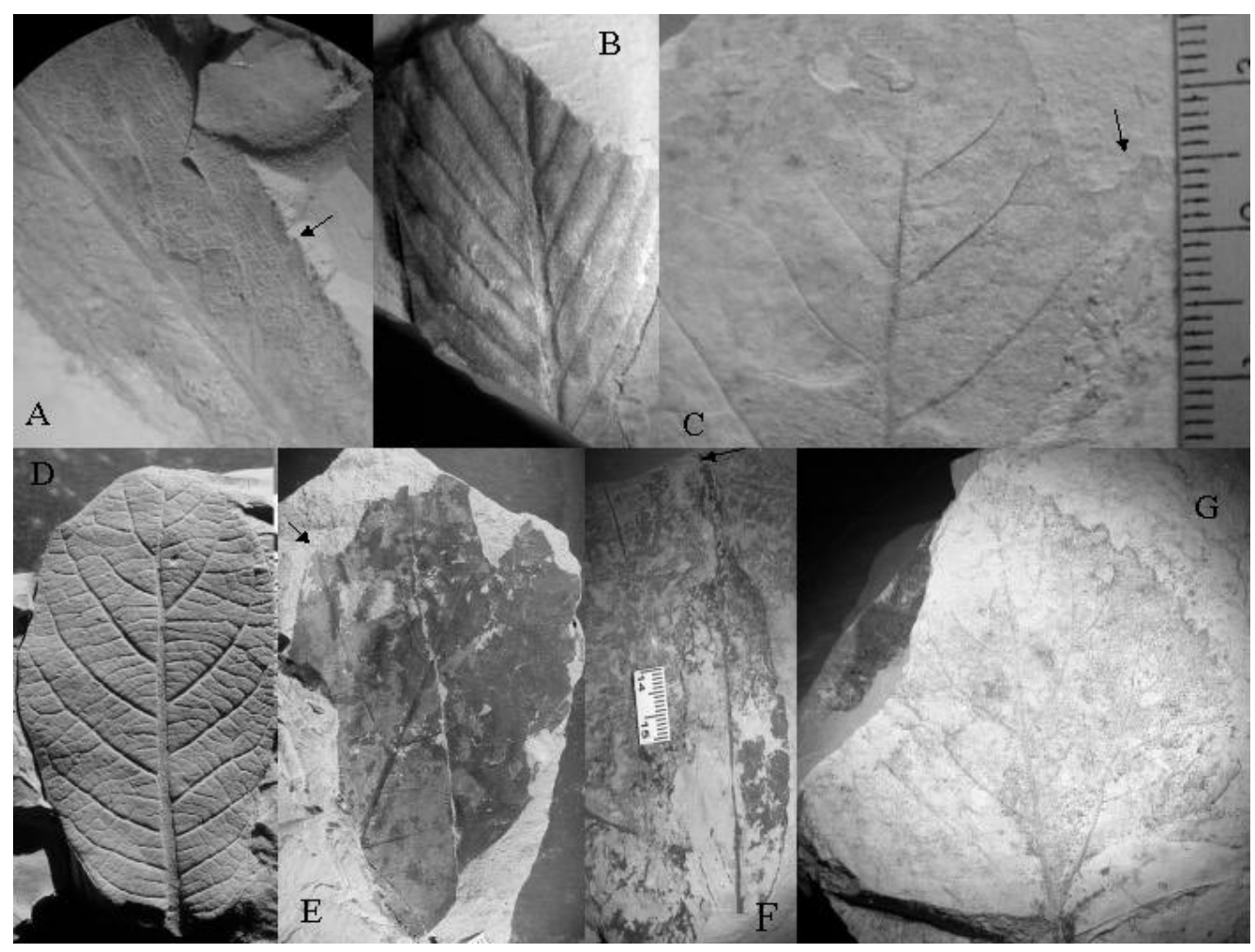

Figura 4. Hojas fósiles presentes en la comunidad de Santa María Amajac. Entre paréntesis se da el número de catálogo: a) Salix sp. (CFZ 93); b) Cercocarpus sp. (CFZ 631), c) Platanus sp. CFZ 140), nótese los lóbulos (flecha), d) Quercus sp. (CFZ 72), e) Quercus sp. (CFZ 172), f) Hoja sin determinar, nótese el ápice agudo (flecha); g) Populus sp. (CFZ 238).

anómalo que pueda provocar la frecuencia alta o baja de un carácter en el conjunto de hojas (Wiemann et al., 1998). En este tipo de análisis la localidad fósil entra como una muestra pasiva, por lo que no altera las correlaciones de las comunidades actuales y los parámetros meteorológicos (Herman y Spicer, 1996).

Los valores de los once parámetros meteorológicos (cuadro 2) son similares a lo indicado cualitativamente por la fisonomía de las hojas. Con base en los valores de TMA, TMC, TMF y PMA, y de acuerdo a la clasificación de Köppen modificada por García (1973) para los climas de la República Mexicana, es posible inferir el clima que se desarrolló durante el Plioceno en la localidad. Éste corresponde a uno templado, ya que los valores de temperatura media anual (entre 12 y $18^{\circ} \mathrm{C}$ ), de temperatura del mes más frío (entre -3 y $\left.18^{\circ} \mathrm{C}\right)$ y de temperatura del mes más cálido $\left(>6.5^{\circ} \mathrm{C}\right) \mathrm{se}$ encuentran en estos intervalos. La diferencia entre TMC y TMF (19.4), indica condiciones muy extremosas. De acuerdo a la precipitación de los tres meses más secos y la precipitación anual, así como el cociente entre PMA y TMA (38.92), se sugiere que el clima fue de tipo subhúmedo (García, 1973), correspondiente a la fórmula climática $\mathrm{Ca}(\mathrm{w})\left(\mathrm{w}_{0}\right)\left(\mathrm{e}^{\prime}\right)$.
Implicaciones de la inferencia paleoclimática. Con la inferencia de las condiciones climáticas de Santa María Amajac durante el Plioceno, podemos denotar dos aspectos que podrían generalizarse para la región: por un lado, conocer las condiciones bajo las cuales se desarrolló la paleocomunidad vegetal, y por el otro, determinar si han ocurrido cambios climáticos.

La composición florística de la paleocomunidad vegetal de Santa María Amajac no se conoce en su totalidad (figura 4, f). Hasta la fecha sólo se han identificado las hojas más abundantes que corresponden a representantes de la familia Platanaceae con el género Platanus (figura 4, c), de las familias Salicaceae y Rosaceae (figura 4, a, b, g) y la familia Fagaceae con el género Quercus (figura 4, d, e) (AguilarArellano y Ortíz-Martínez, 2000; Ortiz-Martínez y VelascoDe León, 2000, Velasco-De León y Aguilar-Arellano, 2000). Al tener el listado florístico completo de la localidad, podremos conocer qué tipo de taxa estaban adaptados a esas condiciones, para así proponer la comunidad vegetal que existió en la zona.

Al comparar los parámetros climáticos actuales de la localidad con los estimados para el Plioceno (cuadro 2), se observa que los parámetros que consideran la 
Cuadro 2. Parámetros meteorológicos esperados y actuales de la localidad de Santa María Amajac. Para el significado de las siglas ver el texto.

\begin{tabular}{ccc}
\hline Parámetros meteorológicos & En el Plioceno & En la actualidad* \\
\hline TMA & $16.7 \pm 1.2^{\circ} \mathrm{C}$ & $16.9^{\circ} \mathrm{C}$ \\
TMC & $26.4 \pm 1.6^{\circ} \mathrm{C}$ & $19.8^{\circ} \mathrm{C}$ \\
TMF & $7{ }^{\circ} \mathrm{C}$ & $13.2^{\circ} \mathrm{C}$ \\
LEC & $9.2 \mathrm{meses}$ & - \\
PMA & $650 \pm 120 \mathrm{~mm}$ & $458.5 \mathrm{~mm}$ \\
PMEC & $72 \mathrm{~mm}$ & - \\
3MH & $280 \mathrm{~mm}$ & $76.3 \mathrm{~mm}$ \\
3MS & $62 \mathrm{~mm}$ & $7.8 \mathrm{~mm}$ \\
HR & $58 \%$ & - \\
HE & $7.8 \mathrm{~g} / \mathrm{kg}$ & - \\
ENT & 309.5 & - \\
Tipo de clima & $\mathrm{Ca}(\mathrm{w})\left(\mathrm{w}_{0}\right)\left(\mathrm{e}^{\prime}\right)$ & $\mathrm{BS}_{1} \mathrm{kw}^{\prime \prime}\left(\mathrm{i}^{\prime}\right) \mathrm{g}$ \\
\hline
\end{tabular}

\footnotetext{
* En la localidad de estudio no hay estación meteorológica, por lo que los datos se tomaron de una población para la que sí estuvieran disponibles, considerando que las altitudes de ambas fueran similares. Los datos corresponden a la población de Actopan, Hidalgo (García, 1973).
}

temperatura no han variado, pero en cambio los de la precipitación sí. Estas diferencias podrían deberse a que durante el Plioceno existió un lago (Salvador-Flores, 2001), el cual sirvió como un cuerpo regulador de la precipitación. Este lago dejó de existir a finales del Plioceno (Salvador-Flores, 2001), con lo que dejó de ser una fuente que proporcionaba humedad a la región. Otro aspecto a considerar son los cambios geológicos que ocurrieron en la región durante el Cenozoico Tardío, caracterizados por eventos volcánicos y de orogenia, lo que contribuiría a que en la actualidad se encuentra muy marcado el fenómeno de la "sombra de lluvia" y con ello la disminución de la humedad (Salvador-Flores, 2001).

Es necesario analizar otras localidades fósiles de plantas y obtener sus datos climáticos, y con ello establecer los cambios que han tenido lugar a través del tiempo, como lo sugiere la evolución geológica de la región (Salvador-Flores, 2001). Además, hace falta estudiar las repercusiones en las plantas, ya que las fluctuaciones del clima no sólo producen nuevas adaptaciones, sino que además provocan cambios muy grandes que pueden llevar a algunos grupos a la extinción y el cambio de distribución en otros (Wolfe, 1993).

Por último, uno de los cuestionamientos de importancia al hacer inferencias paleoclimáticas utilizando el método fisonómico, es si existen correlaciones a nivel mundial entre la morfología foliar y el clima, y cuál puede ser su repercusión en los valores estimados para las localidades fósiles. Stranks y England (1997) consideran que algunos parámetros climáticos pueden mostrar un impacto regional, ya que la morfología de la hoja no depende únicamente de los parámetros climáticos, sino de muchos otros factores.

Aunque CLAMP ha resultado ser una buena base para las estimaciones paleoclimáticas (Wiemann et al., 1998) y permitió inferir el clima de la localidad de estudio, GregoryWodzicki (2000) considera que una buena estimación del paleoclima dependerá de la base de datos que se utilice, ya que puede existir una sobreestimación de los parámetros climáticos al usar los datos de regiones alejadas al sitio en estudio, por lo que recomienda emplear datos de la misma región, es decir, analizar comunidades locales con las fósiles. De México, CLAMP sólo incluye localidades de los estados de Baja California Sur (cinco) y Sonora (siete) (Wolfe, 1993). Por ello, es necesaria la generación de una base de datos para las comunidades vegetales del país que incluya la morfología foliar y los parámetros meteorológicos de la zona, y a partir de éstas establecer un marco de referencia con el cual se puedan hacer inferencias sobre los climas de las comunidades vegetales fósiles de México.

\section{Agradecimientos}

Se agradece a Erika L. Ortíz Martínez su colaboración en las primeras etapas de este trabajo; a Josefa y Alfredo Hernández Gallardo por la ayuda prestada en el trabajo de campo; a Alejandrina Ávila Ortiz, Eloy Solano Camacho, David N. Espinosa Organista, Carlos Pérez Malváez, Oscar J. Polaco y dos revisores anónimos por las observaciones y sugerencias que enriquecieron este escrito. FJA agradece a CONACYT (Becario 162941) y DGEP-UNAM por el apoyo recibido durante la realización de este escrito. 


\section{Literatura Citada}

Aguilar-Arellano F.J. y Ortíz-Martínez E.L. 2000. Estudio paleoecológico de la flora pliocénica de Santa María Amajac, Hidalgo: inferencia del paleoclima y de la paleocomunidad. Tesis de Licenciatura, Facultad de Estudios Superiores Zaragoza, Universidad Nacional Autónoma de México, México, D.F. 62 pp.

Bailey Y.W. y Sinnott E.W. 1915. A botanical index of Cretaceous and Tertiary climates. Science 41:831-834.

Beltrán-Romero F. y Luna-Gómez P. 1994. Estudio geológico de la región de Santa María Amajac, Municipio de Atotonilco el Grande, Estado de Hidalgo. Tesis de Licenciatura, Facultad de Ingeniería, Universidad Nacional Autónoma de México, México, D.F. 131 pp.

Carranza-Castañeda O. y Miller W.E. 1998. Paleofaunas de vertebrados de las cuencas sedimentarias del Terciario Tardío de la faja volcánica transmexicana. En: Carranza-Castañeda O. y Córdoba-Méndez D.A. Eds. Avances en Investigación, Paleontología de Vertebrados, pp. 85-95, Instituto de Investigaciones en Ciencias de la Tierra, Universidad Autónoma del Estado de Hidalgo, Pachuca, Hgo.

Castillo-Cerón J.M. 1994. Bioestratigrafía de los sedimentos continentales del área de Zacualtipán (Mioceno Tardío), estado de Hidalgo, México. Tesis de Maestría, Facultad de Ciencias, Universidad Nacional Autónoma de México, México, D.F. 55 pp.

Castillo-Cerón J.M., Cabral-Perdomo M.A. y Carranza-Castañeda O. 1996. Vertebrados Fósiles del Estado de Hidalgo. Universidad Autónoma del Estado de Hidalgo, Pachuca, Hgo.

Cavalier C., Cháteauneuf J.J., Pomerol C., Rabussier D., Renard M. y Vergnaud-Grazzini C. 1981. The geological events at the Eocene/Oligocene boundary. Palaeogeography, Palaeoclimatology, Palaeoecology 36:223-248.

Daubenmire R.F. 1982. Ecología Vegetal: Tratado de Autoecología de Plantas. 3a. ed. Limusa, México, D.F.

Dilcher D.L. 1973. The Eocene floras of southeastern North America. En: Graham A. Ed. Vegetation and Vegetational History of Northern Latin America, pp. 39-59, Elsevier, Nueva York.

Dolph G.E. y Dilcher D.L. 1979. Foliar physiognomy as an aid in determining paleoclimate. Palaeontographica, Abteilung $B$ 170:151-172.

Freudenberg W. 1922. Die Saügetierfauna des Pliocäns und Postpliocäns von Mexiko: Mastodonten und Elephanten. Geologische und Palaeontologische Abhandlungen 14:103-176.

García E. 1973. Modificaciones al Sistema de Clasificación Climática de Köppen (Para Adaptarlo a las Condiciones de la República Mexicana). 2a. ed., Universidad Nacional Autónoma de México, México, D.F.

García E. 1986. Apuntes de Climatología. 5a. ed., editado por la autora, México, D.F.

González-Gallardo S. 1987. Estado actual del conocimiento de plantas fósiles superiores de México. Tesis de Licenciatura, Escuela Nacional de Estudios Profesionales Iztacala, Universidad Nacional Autónoma de México, Los Reyes Iztacala, Edo. de México, 121 pp.

Gregory-Wodzicki K.M. 2000. Relationships between leaf morphology and climate, Bolivia: implications for estimating paleoclimate from fossil floras. Paleobiology 26: 668-688.
Herman A.B. y Spicer R.A. 1996. Paleobotanical evidence for a warm Cretaceous Arctic Ocean. Nature 380:330-333.

Herman A. B., Spicer R. A., Wolfe J. A. y Collinson M. E. 1996. A guide to obtaining a CLAMP analysis. Manuscrito inédito, 14 pp.

Kovach W.L. y Spicer R.A. 1995. Canonical correspondence analysis of leaf physiognomy: a contributions to the development of a new palaeoclimatological tool. Palaeoclimates 1:125-138.

Kowallis B.J., Swisher C.C., Carranza-Castañeda O., Miller W.E. y Tingey D.G. 1998. Preliminary dates in selected late tertiary vertebrate faunas from Mexico. En: Carranza-Castañeda O. y Córdoba-Méndez D.A. Eds. Avances en Investigación, Paleontología de Vertebrados, pp. 10-35. Instituto de Investigaciones en Ciencias de la Tierra, Universidad Autónoma del Estado de Hidalgo, Pachuca, Hidalgo.

Microsoft Corporation. 1994. EXCEL versión 5.0. Nueva York.

Miller W.E. 1987. Mammut americanum, Utah's first record of the American mastodon. Journal of Paleontology 61:168-183.

Ortiz-Martínez E.L. y Velasco-De León M.P. 2000. Estudio de taxonomía numérica de hojas fósiles de Santa María Amajac del Plioceno del estado de Hidalgo. En: López-Oliva J.G., ArzSola J.A., Cossío-Torres T., De León-Gómez H., Martínez J.L., Medina-Barrera F., Ramírez-Fernández J.A. Eds. VII Congreso Nacional de Paleontología y I Simposio Geológico en el Noreste de México. 22-28 julio 2000. Libro de Resúmenes, pp. 54-55, Universidad Autónoma de Nuevo León, Linares, N.L.

Polaco O.J., Arroyo-Cabrales J., Corona-M.E. y López-Oliva J.G. 2001. The American mastodon (Mammut americanum) in Mexico. En: Cavarretta E., Gioia P., Mussi M. y Palombo M. R. Eds., The World of Elephants. Proceedings of the 1st. International Congress, pp. 237-242, Consiglio Nazionale delle Ricerche, Roma.

Raunkier C. 1934. The Life Forms of Plants and Statistical Plant Geography. Clarendon University Press, Oxford.

Salvador-Flores R. 2001. Origen, sedimentología y estratigrafía del paleolago de Amajac, Hidalgo. Tesis de Licenciatura, Facultad de Ingeniería, Universidad Nacional Autónoma de México, México, D.F. 87 pp.

Sear C.B. y Kelly P.M. 1980. Eruption of Mt. St. Helens: effects on climate. Nature 285:533-535.

Segerstrom, K. 1961. Geología del suroeste del estado de Hidalgo y del noreste del estado de México. Boletín de la Asociación Mexicana de Geólogos Petroleros 13:147-168.

Silva-Pineda A. y Buitrón-S. B.E. 2000. Paleontología Mexicana, Plantas Vasculares Fósiles. Facultad de Ingeniería, Universidad Nacional Autónoma de México, México, D.F.

Spicer R.A. 1981. The sorting and deposition of allochthonous plant material in a modern environment at Silwood Lake, Silwood Park, Berkshire, England. U. S. Geological Survey Professional Paper 1143:1-77.

Spicer R.A., Herman A.B. y Wolfe J.A. 2002. CLAMP. Sitio web: http://tabitha.open.ac.uk/spicer/CLAMP/Clampset1.html

Stranks L. y England P. 1997. The use of a resemblance function in the measurement of climatic parameters from the physiognomy of woody dicotyledons. Palaeogeography, Palaeoclimatology, Palaeoecology 131:15-28.

Ter Braak C.J.F. 1991. CANOCO. versión 3.12. Agricultural Mathematics Group DLO. Wageningen, Holanda.

Velasco-De León M.P. 1999. Estudio paleoecológico de una comunidad del Terciario en el estado de Puebla. Tesis doctoral, 
Facultad de Ciencias, Universidad Nacional Autónoma de México, México, D.F., 120 pp.

Velasco-De León M.P. y Aguilar-Arellano F.J. 2000. Presencia del género Platanus en la flora pliocénica de Santa María Amajac, estado de Hidalgo. En: López-Oliva J.G., Arz-Sola J.A., CossioTorres T., De León-Gómez H., Martínez J.L., Medina-Barrera F., Ramírez-Fernández J.A. Eds. VII Congreso Nacional de Paleontología y I Simposio Geológico en el Noreste de México. 22-28 julio 2000. Libro de Resúmenes, pp. 83-84, Universidad Autónoma de Nuevo León, Linares, N.L.

Villarello J. y Böse E. 1902. Criaderos de fierro de la Hacienda de Vaquerías en el estado de Hidalgo. Boletín del Instituto Geológico Mexicano 16:15-44.

Weber R. y Cevallos-Ferriz S.R.S. 1994. Perfil actual y perspectivas de la paleobotánica en México. Boletín de la Sociedad Botánica de México 55:141-148.

Wiemann W.C., Manchester S.R., Dilcher D.L., Hinojosa L.F. y Wheleer E.A. 1998. Estimation of temperature and precipitation from morphological characters of dicotyledonous leaves. American Journal of Botany 85:1796-1802.

Wilf P. 1997. When are leaves good thermometers?: A new case for leaf margin analysis. Paleobiology 23:373-390.

Wing S.L. y Sues H.D. 1992. Mesozoic and early Cenozoic terrestrial ecosystems. En: Behrensmeyer A.K., Damuth J.D., DiMichele W.A., Potts R., Sues H.D. y Wing S.L. Eds. Terrestrial ecosystems through time: evolutionary paleoecology of terrestrial plants and animals, pp. 327-417, University of Chicago Press, Chicago.

Wolfe J.A. 1969. Paleogene floras from the Gulf of Alaska region. U. S. Geological Survey Open-file Report No. 1268. Menlo Park, California.

Wolfe, J.A. 1971. Tertiary climatic fluctuations and methods of analysis of Tertiary floras. Palaeogeography, Palaeoclimatology, Palaeoecology 9:27-57.

Wolfe J.A. 1993. A method of obtaining climatic parameters from leaf assemblages. U.S. Geological Survey Bulletin 2040:1-71.

Wolfe J.A. 1995. Paleoclimatic estimates from Tertiary leaf assemblages. Annual Review of Earth and Planetary Sciences 23:119-142.

Wolfe J.A. 2001. Using leaves for paleoclimatic estimates. PaleoBios 21:135.

Wolfe J.A. y Hopkins D.M. 1967. Climatic changes recorded by land floras in northwestern North America. En: Hatai K. Ed. Tertiary correlations and climatic changes in the Pacific, pp. 67-76, Symposium of the 11th Pacific Scientific Congress, Tokyo. 
A péndice 1. Caracteres foliares y estados de carácter utilizados en el análisis por medio del método de CLAM P.

El método de CLAM P considera los siguientes caracteres foliares: la modificación de la lámina en lóbulos; el margen; el tipo de diente; el tamaño; el tipo de ápice; el tipo de base; la relación largo-ancho y la forma (Wolfe, 1993; Herman et al., 1996). A continuación se dan los estados usados para cada carácter, en la versión de CLAM P3B (Herman et al., 1996), así como en la versión actual (Spicer et al., 2002) y como son observados en los ejemplares. Asimismo se presenta el número que le corresponde en la base de datos.

Hoja con lóbulos (1): se considera la presencia de hojas con lóbulos, que puede presentarse ya sea palmadoIobado, e.g. algunas hojas de Acer (figura 3, a), o pinado-lobado, e.g. algunas hojas de $Q$ uercus (figura 3, b).

El margen (2): se observa si existe modificación en el margen de la lámina; considera dos estados, el entero y el dentado (figura 3, c-d).

Tipo de diente: si el margen es dentado, se observa la forma de los dientes y el espacio entre cada uno. CLAM P considera cinco estados: regulares (3), cuando la longitud del espacio de los costados de dos dientes adyacentes es al menos de una tercera parte (figura 3, e); cerrado (4), los costados basales de los dientes no son más largos que tres veces los costados apicales (figura $3, \mathrm{f}$ ); redondo $\mathbf{y} / \mathbf{0}$ apresado (5), es redondo cuando el diente tiene un costado apical y uno basal convexo, el cual se extiende hasta la punta (figura $3, \mathrm{~g}$ ), es apresado, cuando el flanco apical del diente es cóncavo y el basal convexo, curvándose hacia la lámina (figura 3, h); agudo (6), los costados apical y basal del diente forman una punta (figura 3, i); compuestos (7), el diente tiene dientes pequeños en su costado basal (figura $3, \mathrm{j})$.

Tamaño: a diferencia de CLAM P, que emplea una plantilla para determinar el tamaño de la hoja (Wolfe, 1993; Spicer et al., 2002), se utilizó la longitud de la hoja tomada desde la base hasta el ápice, y se definieron intervalos siguiendo los estados que considera CLAMP, que son nueve: nanófilo (8), $<1 \mathrm{~cm}$; leptófilo $1(\mathbf{9}), 1 \mathrm{~cm}$; leptófilo 2 (10), 1.1-2 cm; micrófilo 1 (11), 2.1-4.2 cm; micrófilo 2 (12), 4.3-7.2 cm; micrófilo 3 (13), 7.3-12cm; mesófilo 1 (14), 12.1-16.1 cm; mesófilo 2 (15), 16.2-30 cm, y mesófilo 3 (16), $>30 \mathrm{~cm}$.

Tipo de ápice: considera cuatro estados: emarginado (17), la parte más apical de la lámina forma una curva convexa hacia su base (figura 3, k); redondo (18), la cuarta parte más apical de la lámina tiene un margen que se curva convexamente y los dos lados forman una curva (figura 3, I); agudo (19), la cuarta parte más apical de la lámina tiene un margen que es recto y los dos lados forman una punta (figura 3, m-n); atenuado (20), la cuarta parte más apical de la lámina y el margen de ambos lados cambia de uno lineal o convexo a un margen cóncavo, y éste se extiende en una distancia de al menos $1 \mathrm{~cm}$ (figura 3, o-p).

Tipo de base: tiene tres estados: cordado (21), la parte basal de la lámina se extiende basalmente más allá de su unión con el pecíolo (figura 3, q-r); redonda (22), más de la cuarta parte basal de la lámina tiene un margen convexo (figura 3, s-t); agudo (23), más de la cuarta parte basal de la lámina tiene un margen recto o cóncavo (figura 3, u-v).

Relación largo-ancho (L:W): se utiliza la longitud y el ancho de la hoja, este último se toma en la parte más amplia de la lámina. Se consideran cinco estados: >1:1 (24), 1-2:1 (25), 2-3:1 (26), 3-4:1 (27), >4:1 (28).

Forma: se considera la parte más ancha de la lámina. CLAM P registra tres estados: obovada (29), localizada en más de la tercera parte apical de la lámina (figura 3, w); elíptica (30), se encuentra a la mitad de la lámina (figura 3 , X); ovada (31), ubicada en más de la tercera parte basal de la lámina (figura 3, y).

La base de CLAM P es de tipo cuantitativo y lo que refleja es el porcentaje de representación de cada estado de carácter en la comunidad vegetal. Para obtenerlo, primero se codifican los estados de carácter en términos numéricos, lo cual varía dependiendo del carácter. En el caso de las hojas lobadas y el tipo de margen, los estados de carácter se codifican de la siguiente manera, si se encuentran presentes (entero, en caso del margen) en toda la muestra reciben un registro de 1, 0.5 si no están presentes en toda la muestra y 0 si están ausentes (lo que equivale a la presencia de dientes en el margen); esta misma codificación se utiliza para el ápice emarginado. Para los siguientes caracteres, se considera que sus estados de carácter suman un total de 1, por lo que si está presente un solo estado de carácter recibe un registro de 1 , pero si hay más estados el valor de la unidad se divide entre el número total de estados de carácter presentes en la categoría (e.g., si hay dos estados, cada uno recibe 0.5; si hay tres, 0.33 en cada uno, y así sucesivamente).

Para la obtención del porcentaje de cada estado de carácter, se suman los registros por estado de carácter, se divide el resultado entre el número total de especies o morfotipos presentes en la localidad de estudio, y se multiplica por 100. En el caso de los fósiles, la suma se divide entre el número de morfotipos que presentan el carácter, ya que no siempre en los fósiles se preservan todos (Wolfe, 1993; Herman et al., 1996). El porcentaje se da en números enteros, por lo que las fracciones se redondean: 0.5 ó más sube al siguiente entero, y 0.499 o menos baja al siguiente.

En el siguiente cuadro se presenta el registro de los 31 estados de carácter de los 39 morfotipos distinguidos en Santa M aría Amajac. El último renglón del cuadro corresponde a los porcentajes de cada estado de carácter que se anexaron a la base de CLAM P3B. 
Las columnas corresponden a los estados de carácter, los renglones a cada tipo de hoja.

\begin{tabular}{|c|c|c|c|c|c|c|c|c|c|c|c|c|c|c|c|c|c|c|c|c|c|c|c|c|c|c|c|c|c|c|c|}
\hline & 1 & 2 & 3 & 4 & 5 & 6 & 7 & 8 & 9 & 10 & 11 & 12 & 13 & 14 & 15 & 16 & 17 & 18 & 19 & 20 & 21 & 22 & 23 & 24 & 25 & 26 & 27 & 28 & 29 & 30 & 31 \\
\hline 1 & 1 & 1 & 0 & 0 & 0 & 0 & 0 & - & - & _. & - & - & 1 & _ & - & - & _ & - & - & - & _ & - & 1 & - & 1 & - & - & _ & - & - & 1 \\
\hline 2 & 0 & 1 & 0 & 0 & 0 & 0 & 0 & - & - & - & - & 1 & - & 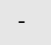 & - & - & - & 1 & . & 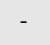 & 1 & 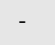 & - & - & 1 & - & - & 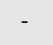 & 1 & 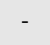 & 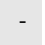 \\
\hline 3 & 0 & 0 & 1 & 0 & 0 & 0 & 0 & 1 & _ & - & - & - & - & - & - & - & - & - & - & - & 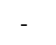 & 1 & - & - & - & 1 & - & - & - & - & 1 \\
\hline 4 & 0 & 1 & 0 & 0 & 0 & 0 & 0 & 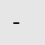 & - & - & 1 & - & - & - & - & - & - & 1 & - & - & 1 & - & - & - & - & 1 & - & 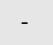 & - & 1 & - \\
\hline 5 & 0 & 1 & 0 & 0 & 0 & 0 & 0 & - & - & - & 1 & - & - & - & - & - & - & - & 1 & - & 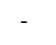 & - & 1 & - & 1 & - & - & - & - & - & 1 \\
\hline 6 & 0 & 1 & 0 & 0 & 0 & 0 & 0 & - & - & - & - & 1 & - & - & - & - & - & - & 1 & - & - & - & 1 & - & 1 & - & - & - & - & 1 & - \\
\hline 7 & 0 & 0 & 1 & 0 & 0 & 0 & 0 & - & - & - & 1 & - & - & - & - & - & - & - & - & - & - & 1 & - & - & - & 1 & - & - & - & - & 1 \\
\hline 8 & 0 & 0 & 1 & 0 & 0 & 0 & 0 & - & - & - & 1 & - & - & - & - & - & - & - & 1 & - & - & 1 & - & - & 1 & - & - & - & - & - & 1 \\
\hline 9 & 0 & 1 & 0 & 0 & 0 & 0 & 0 & - & - & - & 1 & - & - & - & - & - & - & 1 & - & - & - & - & 1 & 1 & - & - & - & - & - & - & 1 \\
\hline 10 & 0 & 1 & 0 & 0 & 0 & 0 & 0 & - & - & - & 1 & - & - & - & - & - & - & - & 1 & - & - & 1 & - & - & 1 & - & - & - & - & 1 & - \\
\hline 11 & 0 & 1 & 0 & 0 & 0 & 0 & 0 & - & - & - & 1 & - & - & - & - & - & - & - & - & - & - & - & 1 & - & 1 & - & - & - & 1 & - & - \\
\hline 12 & 0 & 1 & 0 & 0 & 0 & 0 & 0 & - & - & - & - & 1 & - & - & - & - & - & - & 1 & - & - & 1 & - & - & 1 & - & - & - & - & - & 1 \\
\hline 13 & 0 & 1 & 0 & 0 & 0 & 0 & 0 & - & - & 1 & - & - & - & - & - & - & - & - & - & - & - & 1 & - & - & 1 & - & - & - & 1 & - & - \\
\hline 14 & 0 & 1 & 0 & 0 & 0 & 0 & 0 & - & - & - & 1 & - & - & - & - & - & - & - & 1 & - & - & - & 1 & - & 1 & - & - & - & - & 1 & - \\
\hline 15 & 0 & 1 & 0 & 0 & 0 & 0 & 0 & - & - & 1 & - & - & - & - & - & - & - & 1 & - & - & - & - & - & - & 1 & - & - & - & - & 1 & - \\
\hline 16 & 0 & 1 & 0 & 0 & 0 & 0 & 0 & - & - & - & - & - & 1 & - & - & - & - & 1 & - & - & - & 1 & - & - & 1 & - & - & - & - & - & 1 \\
\hline 17 & 0 & 1 & 0 & 0 & 0 & 0 & 0 & - & - & - & 1 & - & - & - & - & - & - & - & 1 & - & - & - & 1 & - & 1 & - & - & - & - & - & 1 \\
\hline 18 & 0 & 0 & 1 & 0 & 0 & 0 & 0 & - & - & - & 1 & - & - & - & - & - & - & - & 1 & - & - & - & 1 & - & 1 & - & - & - & - & - & 1 \\
\hline 19 & 0 & 1 & 0 & 0 & 0 & 0 & 0 & - & - & - & - & 1 & - & - & - & - & - & 1 & - & - & - & 1 & - & - & 1 & - & - & - & - & 1 & - \\
\hline 20 & 0 & 1 & 0 & 0 & 0 & 0 & 0 & - & - & - & - & 1 & - & - & - & - & - & - & - & - & - & 1 & - & - & - & - & - & 1 & - & 1 & - \\
\hline 21 & 0 & 0 & 1 & 0 & 0 & 0 & 0 & - & - & - & 1 & - & - & - & - & - & - & - & 1 & - & - & 1 & - & - & 1 & - & - & - & - & 1 & - \\
\hline 22 & 0 & 0 & 1 & 0 & 0 & 0 & 0 & - & - & - & - & 1 & - & - & - & - & - & - & 1 & - & - & - & - & - & 1 & - & - & - & - & 1 & - \\
\hline 23 & 0 & 0 & 1 & 0 & 0 & 0 & 0 & 1 & - & - & - & - & - & - & - & - & - & 1 & - & - & - & - & 1 & - & 1 & - & - & - & 1 & - & - \\
\hline 24 & 0 & 0 & 1 & 0 & 0 & 0 & 0 & - & - & - & 1 & - & - & - & - & - & - & - & - & - & - & 1 & - & 1 & - & - & - & - & 1 & - & - \\
\hline 25 & 0 & 1 & 0 & 0 & 0 & 0 & 0 & - & - & - & 1 & - & - & - & - & - & - & - & 1 & - & - & - & 1 & - & 1 & - & - & - & - & 1 & - \\
\hline 26 & 0 & 1 & 0 & 0 & 0 & 0 & 0 & - & - & - & 1 & - & - & - & - & - & - & - & 1 & - & - & - & 1 & - & - & 1 & - & - & - & 1 & - \\
\hline 27 & 0 & 1 & 0 & 0 & 0 & 0 & 0 & - & - & - & 1 & - & - & - & - & - & - & - & 1 & - & - & - & 1 & - & 1 & - & - & - & - & 1 & - \\
\hline 28 & 0 & 1 & 0 & 0 & 0 & 0 & 0 & - & - & - & - & 1 & - & - & - & - & - & 1 & - & - & - & 1 & - & - & 1 & 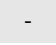 & - & 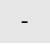 & - & 1 & - \\
\hline 29 & 0 & 1 & 0 & 0 & 0 & 0 & 0 & - & - & - & 1 & - & - & - & - & - & - & - & 1 & - & - & 1 & - & - & 1 & - & - & - & - & - & 1 \\
\hline 30 & 0 & 1 & 0 & 0 & 0 & 0 & 0 & - & - & - & - & 1 & - & - & - & - & - & 1 & - & - & - & - & 1 & - & 1 & - & - & - & - & 1 & - \\
\hline 31 & 0 & 1 & 0 & 0 & 0 & 0 & 0 & - & - & - & 1 & - & - & - & - & - & - & - & - & - & - & - & 1 & - & - & - & - & 1 & - & 1 & - \\
\hline 32 & 0 & 0 & 1 & 0 & 0 & 0 & 0 & - & - & - & 1 & - & - & - & - & - & - & - & 1 & - & - & 1 & - & - & 1 & - & - & - & - & - & 1 \\
\hline 33 & 0 & 0 & 1 & 0 & 0 & 0 & 0 & - & 1 & - & - & 1 & - & - & - & - & - & - & 1 & - & - & 1 & - & - & - & 1 & - & - & - & 1 & - \\
\hline 34 & 0 & 0 & 1 & 0 & 0 & 0 & 0 & - & - & - & 1 & - & - & - & - & - & - & 1 & - & - & - & - & 1 & - & 1 & - & - & - & - & - & 1 \\
\hline 35 & 0 & 0 & 1 & 0 & 0 & 0 & 0 & - & - & - & 1 & - & - & - & - & - & - & - & 1 & - & - & - & 1 & - & 1 & - & - & - & - & 1 & - \\
\hline 36 & 0 & 1 & 0 & 0 & 0 & 0 & 0 & . & - & - & 1 & - & - & - & - & - & - & - & 1 & - & - & - & 1 & - & 1 & - & - & - & - & 1 & - \\
\hline 37 & 0 & 1 & 0 & 0 & 0 & 0 & 0 & - & - & - & 1 & - & - & - & - & - & - & - & 1 & - & - & - & 1 & - & 1 & - & - & - & - & 1 & - \\
\hline 38 & 0 & 0 & 1 & 0 & 0 & 0 & 0 & - & - & - & - & 1 & - & - & - & - & - & - & 1 & - & - & - & 1 & - & 1 & - & - & - & - & 1 & - \\
\hline 39 & 0 & 0 & 1 & 0 & 0 & 0 & 0 & - & - & - & - & 1 & - & - & - & - & - & - & 1 & - & - & - & 1 & - & - & 1 & - & - & - & 1 & - \\
\hline Total & 1 & 28 & 11 & 0 & 0 & 0 & 0 & 2 & 1 & 2 & 22 & 10 & 2 & 0 & 0 & 0 & 0 & 10 & 21 & 0 & 2 & 15 & 20 & 2 & 29 & 6 & 0 & 2 & 5 & 21 & 13 \\
\hline$\%$ & 3 & 72 & 28 & 0 & 0 & 0 & 0 & 5 & 3 & 5 & 56 & 26 & 5 & 0 & 0 & 0 & 0 & 32 & 68 & 0 & 6 & 40 & 54 & 5 & 74 & 15 & 0 & 5 & 13 & 54 & 33 \\
\hline
\end{tabular}

